\title{
A COMPREHENSIVE REVIEW ON DIGITAL TWINS FOR SMART ENERGY MANAGEMENT SYSTEM
}

\author{
MARIO LAMAGNA, DANIELE GROPPI, MEYSAM M. NEZHAD \& GIUSEPPE PIRAS \\ Department of Astronautic, Electrical and Energy Engineering of Sapienza University of Rome, Italy.
}

\begin{abstract}
Energy systems digitalisation represents the energy sector's future, and Digital Twins represent the most advanced and complete way to monitor and optimally manage a complex system such as the upcoming solutions. Those latter will comprehend several energy generators, traditional and/or from renewable energy sources (RESs), different energy storage systems using several energy vectors and that interconnect different energy-consuming sectors (power, thermal, transport sectors) and that fully exploit the potential synergies offered by such interconnected system. Nevertheless, since the first conceptualisation of digital twins in the first years of the 21 st century, its use has not started yet for different reasons that are affecting the adoption of this game-changer approach. Hence, what are the main barriers that are holding back the adoption of digital twins in smart energy systems? The present review paper answers this research question while discussing the case studies that can be found in literature and analysing the different approaches and the system architectures that have been tested or simply idealised. This paper provides a basis for future research that aims at applying the digital twin concept in the energy sector and particularly for power grid management. It deals with the challenges of big data management, the ones related to real-time measurements and continuous communication between the real-world system and its digital twin, the investment for measuring systems, the issues connected with the use of large data centres and the correlated energy-related challenges and doubts. The review analyses the challenges that have been encountered so far, the proposed solutions and the opportunities that such a 'work in progress' topic offers.
\end{abstract}

Keywords: barriers, digital twin, energy systems, modelling, real-time analyses.

\section{INTRODUCTION}

Today's energy system architecture is turning toward a massive electrification of consumptions and renewable energy source (RES) penetration [1]. In this regard, it is key to rely on technologies able to monitor and coordinate the energy fluxes, expressed in terms of demand and production. In the real world, this technology is translated into a series of sensors installed in the system that feed databases and algorithms dedicated to analysing the different fluctuations at any time. Furthermore, this is also coupled with the ability to predict the possible variations in future conditions, so as to be able to promptly adapt the operating technologies accordingly [2].

The digital twin (DT) concept, introduced by Micheal Grieves, reflects this idea which is fundamental to support the future energy system [3]. According to Grieves, the DT system is based on three concepts: physical products in real space, virtual products in virtual space and the connections of data and information that connect the physical and virtual space [4].

Such a system will be able to mirror the behaviour of the object in the real environment. The data availability assures a high-fidelity model, and by combining all of this information, compared also with the history data, the DT can forecast and predict the system response in different possible future scenarios [5]. The prediction is fundamental to maintain our system working efficiently and in safe conditions. Additionally, there are also hybrid DT versions where parts of the parameters are not evaluated by sensors; instead, they come directly from the model itself.

The operators working in the energy field understand the potential offered by this approach. For instance, although the investment in electricity grids has decreased by $7 \%$ from 2018 , 


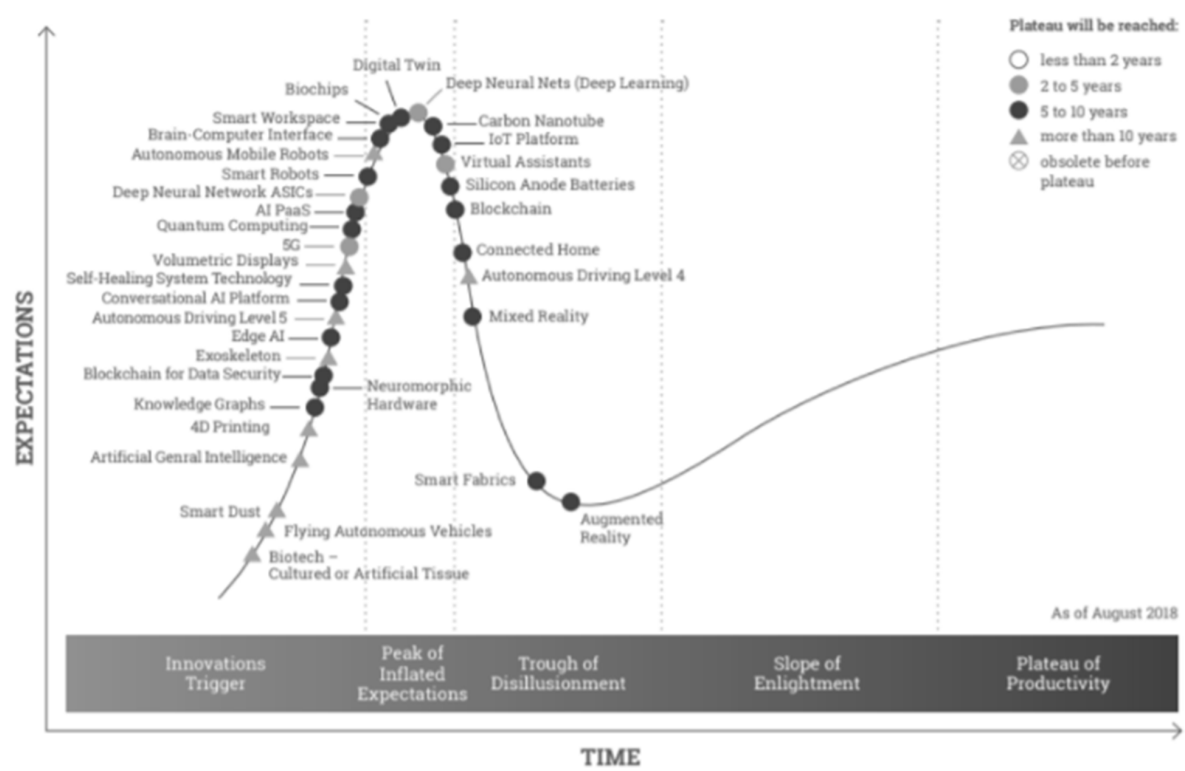

Figure 1: Hype cycle (source: Gartner 2018 [8]).

investment in digital twinning technologies, including the required advanced metering infrastructure, utility automation and artificial intelligence, made up more than $15 \%$ of total grid spending [6].

Digital technologies are arising and develop faster than other technologies used in the power system [7]. Gartner has plotted the key digital technologies in the hype-cycle curve, reported in Fig. 1, to enable an assessment of the expectations of these technologies [8].

Although substation automation has been a trend in recent years [9], in 2019, utilities expanded the use of software platforms to monitor and control them, notably through DTs. National grid operators partnered with data management utilities and sensor manufacturers to create grid and energy systems twins, mapping power flow, voltage and infrastructure from substations to homes [10]. The American Electric Power Authority also announced the digital twinning of its transmission infrastructure [11]. From Gartner work, the high expectations placed on DT technologies are clear. Nevertheless, it is expected that they could reach a satisfactory level of productivity and common use in our everyday life only in a period varying from 5 to 10 years from 2018 [8]. The scope of this review is to analyse the possible barriers hampering the DT technologies to reach their productivity plateau and, at the same time, illustrate the positive results already obtained. In so doing, particular attention will be paid to the energy sector as a whole and energy management systems and power grids that have to communicate and interoperate with homes and transport. The review discussion will be organised in five sections, concerning five different but nevertheless complementary aspects:

- Functioning/operative technologies

- Power grid

- Energy systems

- Buildings

- Transport 


\section{DISCUSSION}

In this paragraph, the five sections will be dealt with in specific chapters. Each of those has been analysed in terms of development and encountered barriers.

\subsection{Functioning/operative technologies}

The DT technology is considered the optimal solution for the control of smart energy systems (SESs). SES are those systems that comprise different energy vectors, several RES generators and storage technologies and most importantly exploit the opportunities offered by sector coupling and demand response (DR) strategies [12]. Many studies have proven that this solution allows better exploitation of non-dispatchable RES [13]. The most analysed sectors are the heating sector and the transportation one that are experiencing the fastest electrification process. This enables them to grow their demand response potential and also the impact on the power grid in terms of higher peak loads. In this framework, there is an extraordinary amount of data to gather, process and analyse to properly control SESs. The DT approach is the best candidate for grasping such complicated problems.

Indeed, a DT is able to:

- Monitor the physical system: through on-field sensors and external sources. All the data necessary for the next steps are called digital thread [14].

- Analyse and plan: this is done thanks to the digital model of the real system and several algorithms and techniques to identify the optimal measures to control the physical system.

- Execute/control the real system: this is done through physical assets (i.e. controllers).

In order to realise it, a DT entails different levels, the physical assets (i.e. sensors and controllers) that have to be connected with digital assets (often cloud-based) in order to store the data, process and analyse them and then simulate the real system behaviour through the digital model in order to find the optimal control strategy that will then be fulfilled by the controllers [15].

The management of such an intricate network is challenging for several reasons such as communication protocols, real-time processes and analyses, security and privacy and all the challenges that are linked to big data (e.g. management, storage, privacy and security). In [16], an edge computing architecture is proposed to overcome such difficulties given their ability to manage heterogeneous data. The proposed system can be divided in three layers: IoT and sensors for data gathering and physical control of the real assets, edge nodes for data processing and computing, and cloud services for statistical analysis and visualisation of data and all operations linked to artificial intelligence.

As said, the main objective of an energy management system is to control all the participating assets optimising different parameters while ensuring several constraints. If the overall objective can be always considered the least economic expenses, each subsystem requires different specific constraints. The power sector must always consider the constraints represented by the power grid in terms of stability and security of supply while also considering the technical characteristics of generators, both traditional and renewable, storage systems and loads. Regarding the thermal sector (i.e. heating and cooling), aside from the constraints connected to the technical characteristics of the thermal generators, the major constraints are connected to the indoor environmental quality that has to be ensured at all times. A similar constraint is necessary for the transport sector when considering electric vehicles (EVs). The power grid must always ensure a proper state of charge when the vehicle is supposed to 


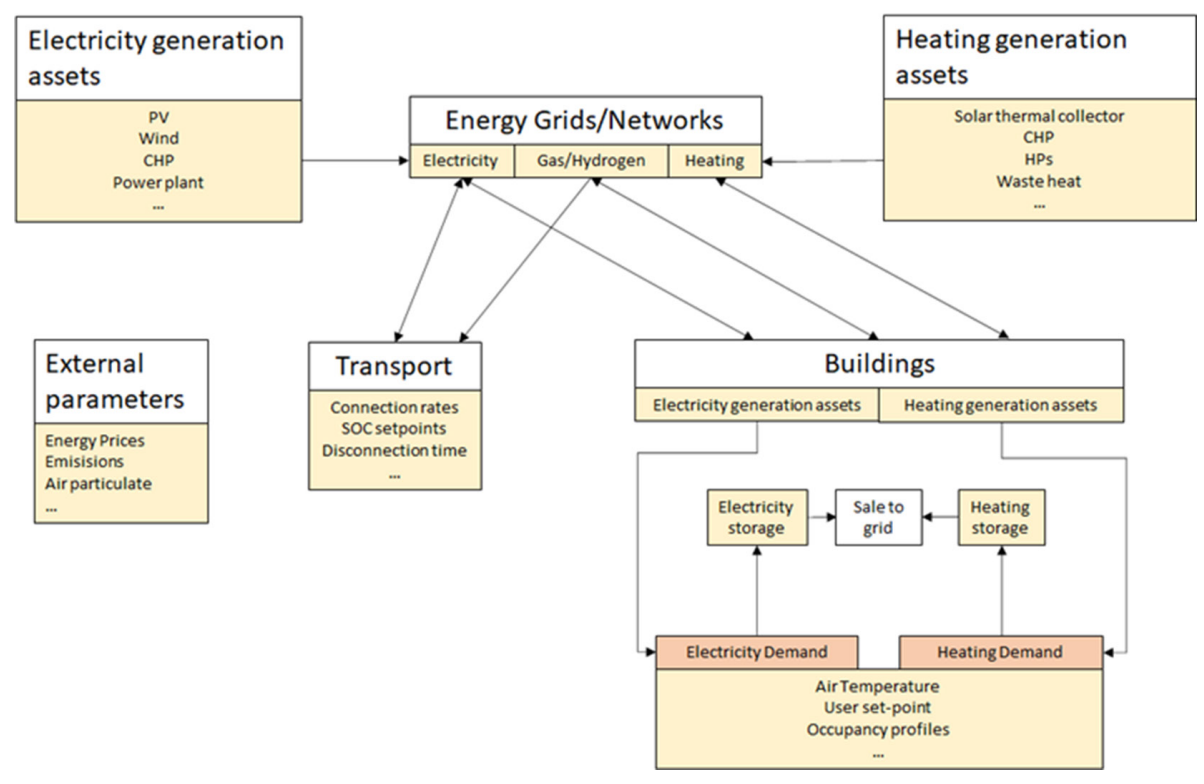

Figure 2: DT synoptic.

detach from the grid (each vehicle will require a different state of charge and different time of connection). Also, it should be considered that several other data, external to the energy system, are necessary to properly analyse the optimal management strategy (e.g. electricity price, gas price, emission indicators and weather data) [15]. A general architecture of such DT is shown in Fig. 2.

The advantage offered by the DT is the possibility to constantly monitor the system with the appropriate sensors and communication. This enables the DT to check that the real system reacted to an input as expected; otherwise the control system will automatically order a different control strategy. Such a difference between the expected result and the obtained one in a perfect model could be attributed to a change in the real-world system. The additional advantage offered by DT is that the model will automatically analyse the reasons that lead to the deviations and will recalibrate itself. This is particularly important in an energy system that entails different sectors and an uncountable number of devices. Indeed, the installation of new charging stations, or heat pumps, or simply the modification of efficiency of some assets might modify the overall system response to a certain control manoeuvre. Thus, the ability of the digital model to modify its parameters to always be updated and able to mirror the real system is priceless.

Even additional challenges and barriers are found when transferring the aforementioned concepts in a business-oriented framework. This is true, for instance, in the case of demand response markets that involve several flexibility providers.

\subsection{The power grid}

National or local power grids offer a perfect field of application to the twinning technology regardless of their sizes. 
The DT framework in the context of the Chinese national power grid was analysed in [17], and a new online analysis digital twin (OADT) was presented to solve real-world complex system problems in large scale (40k+ buses). The results presented in the paper show that the OADT can track the operation state of a large-scale power grid in real time with only a sub-second delay. The only impediment found for the DT application is inferable to software development problems, which needs an exhaustive software platform to be supported. However, the recent information technology advances enable more feasible and practical DT implementation. Similarly, in [18], the DT methodology was applied to understanding the power system dynamics in a way to set the grid to work at the optimum active and reactive settings, by adjusting the voltage stability which is reflected in an overall system reliability increase. The final objective of this research was to demonstrate that thanks to this approach, it is possible to integrate more variable RES technologies (in this case PV panels) in the actual grid, avoiding dangerous voltage variations. The proposed approach was successful, and the only limitation seems to be represented by a limited number of measurement devices installed on the grid.

Similarly, the objective in [19] was to apply the DT to the offshore wind farms transmission systems. The related voltage stability problems can become even a more challenging issue in these conditions. The paper proposed a direct voltage control (DVC) strategy in a real-time environment to stabilise voltage and frequency oscillations by means of a DT model. Moreover, the converters' representation offers a better depiction of the real-world operation.

Reducing the research perimeter, in [20], the attention was focused on distribution network (DN) and the need to create an adequate interface able to gather all the information coming from sensors. Additionally, the authors in [21, 22] focused their work on the DN issues. Their objective was to increase the flexibility of the grid connecting both the local network and the regional power system. In these papers, the DT concept was used to modify the DN planning in execution, individualising in real time the future performance ratings and weak points according to the proposed changes. To reconstruct the virtual model, real-time operating data were assessed and used to build the DT algorithms. In conclusion, this work did not directly analyse the ongoing performances of the grid. Instead, forecasting the possible performances, it proposed different planning adjustments, supporting the applicability of the DT already from the project early stages. This approach is further investigated in [22], where the concept was enlarged by the authors to the distributed production penetration, to help the operators in making better operational decisions. Nevertheless, the presence of massive measuring instrumentation and other devices, many of those accessible by remote control, expose the power grid management to cyberattacks risk. In this regards, in [23], the authors proposed an innovative solution to cope with this problem, a solution based on a IoT-DT model of the cyber-physical system that interacts with the control system to ensure its proper operation. The obtained results demonstrated how the proposed IoT-DT model is able to mitigate a cyberattack simulated as a coordinated false data injection or as an attempted normal service interruption.

\subsection{Energy systems}

DT is a key aspect to increase the efficiency and the management of the energy systems. Fast response and analysis are required while working with electricity independently if the focus is on a transmission grid or a domestic system. Regarding affirmed technologies, most of the research prefers to analyse the entire system instead of a single one. For instance in [24], the analysis was focused on the entire city's energy systems. In this latter research, the energy 
systems were extended to the buildings energy performance, underlying the applicability and the possible interconnectivity with different topics such the Building Information Modelling (BIM) as it will be presented in the next section. Also, in [25], the energy systems are converted in the DT model to recreate the smart grid to be analysed. Differently, when analysing new emerging technologies, the research perimeter remains closer to the system level. For example, hydrogen is confirming its role as enabling technology, representing a promising mechanism to realise sector coupling [26]. Following the other energy system technologies, also hydrogen solutions are experimenting with an exploration phase with DT models. At the moment, it was not possible to find works related to the DT applied to a fuel cell or electrolyser operating in a real context; instead, since the hydrogen topic is still gaining momentum, it was easier to find work related to the performance analysis at lab scale. The most researched aspects are related to the manufacturing and the advancement of this technology. To cope with this technical issue, surrogate modelling methods that combine a state-of-the-art three-dimensional physical model and a data-driven model are investigated in different researches. The result obtained in [27], concerning the DT of a proton exchange membrane fuel cell, can predict its outputs with a root-mean-square error from $3.88 \%$ to $24.80 \%$. This architecture is used to control the healthy operation envelope and state map. Similarly, in [28], a DT model was made for a solid oxide fuel cell (SOFC), starting from a $1 \mathrm{~kW} \mathrm{SOFC}$ data were used to regress the parameters to scale-up the model to $25 \mathrm{~kW}$. The final obtained DT was validated by steady-state data and applied to on-site operation prediction with very high accuracy. The scope of the project was to help operators in the operation strategies selection, by means of simulations to execute the process safely and stably. Being relatively new as a subject, also the manufacturing itself is being investigated to lower initial costs and enhance the manufacturing process, in this regard it could be useful to analyse the information reported in [29] where an exhaustive review on DT-based sustainable intelligent manufacturing is presented.

\subsection{Buildings}

Buildings account for a large portion of the overall energy consumption, and this is divided into electricity and thermal demand that are further split in heating and cooling. As for cooling, this is mostly translated into electricity since the most used technology is heat pumps. Regarding heating instead, the framework is more varied since this can be supplied not only through several different means, such as gas, biomass, and other fossil fuels, but also through electricity thanks to heat pumps (also electric boiler but mostly for hot water instead than indoor ambient heating). Indeed, the energy intensity of buildings also varies depending on the end-use. Commercial buildings will have a much higher energy consumption compared to residential. Given buildings' important role in the energy system, a SES must consider their role in the system both as consumers and service providers, particularly for flexibility services through demand response programmes. For these reasons as well as for a better monitoring and understanding of the energy system, smart metering and IoT devices presence in the building sector have been proliferating in the last years and are expected to keep growing in the years to come. This enables the application of DT concepts in the building sector, and several studies can be found in literature with different objectives and focus.

In [30], the authors apply a DT framework with the aim of monitoring several parameters and, through a serious game, reduce energy consumption and proving that a $40 \%$ energy 
saving can be achieved. This is an example of different services that can be offered by means of DT structure and architecture.

In [31], the authors adopt a DT approach in order to monitor buildings performance as well as renewable energy generation in order to improve overall performance of the smart city by means of an optimal scheduling. They classify home devices in three categories depending on their flexibility, such as shiftable (e.g. dryer), interruptible (e.g. hot water) and weather based (e.g. HVAC).

In [32], the authors presented a framework for near real-time management DR in SES which is able to fully exploit the potential offered by internet of things by means of a software-in-the-loop strategy. They proved the benefits that could be provided to the grid by means of DR strategies at building level. Nevertheless, it is interesting to notice that the term 'digital twin' is never mentioned in the whole article.

Zhou and Zhang [33] focused on a key feature of a DT control system that is the demand forecasting. Particularly, they proposed a model for smart homes based on edge computing that uses the cloud for data processing and for further analysis. The input data are collected both by sensors (i.e. IoT devices) and external/environmental data.

In [34], authors analyse a DT based on machine learning coupled with an urban-scale EnergyPLUS model with the aim of analysing the demand response potential of buildings to provide flexibility services to the grid.

In [35], the authors analyse the importance of edge computing for smart buildings and propose an approach based on the CAFCLA framework (Context-Aware Framework for Collaborative Learning Activities).

In [36], the authors apply a DT approach based on BIM and GIS on the case study in Rome, Italy. The proposed DT model is useful both in the design phase and in the operation phase for optimal management and control thanks to the use of artificial intelligence. The authors conclude that the proposed system is able to optimally modulate loads increasing self-consumption from RESs and decreasing the overall energy consumption.

Particular attention should be paid to the use of BIM in DTs, a phenomenon that has been growing and organically developing in the last period taking on the lesson learned in the manufacturing sector [37]. A remarkable example is provided by Shahinmoghadam et al. [38]; the authors develop a BIM-IoT-based DT that also makes use of virtual reality for thermal and comfort monitoring. In [39], Desogus et al. analysed the use of BIM and IoT in a DT framework for monitoring of indoor conditions (not only temperature and comfort but also luminance, etc.) and of energy consumption. A further trend is that of adopting geo-referencing in parallel to BIM and IoT for an all-inclusive DT able to integrate spatial data for a better visualisation and consequently a better decision making as presented in [40]. Here, the authors develop a common approach for geo-referencing in the framework of GIS/BIM in Industry Foundation Classes (IFCs) format. Also in [41], the topic of integrating GIS and BIM models is analysed. Specifically, the difficulties that lie in the intrinsic differences between the two frameworks that make it complex to convert data into one format to another. Particularly, the authors developed a method to easily convert IFCs into shapefiles in an efficient and reliable way.

\subsection{Transport}

Transport solutions can be associated with moving energy management systems, where the energy source is transformed in motion. Additionally, the recent processes in the transport sectors of electrification and digitalisation are opening interesting new scenarios for the entire 
industry. The DT approach integration in this sector has been addressed by many authors with different focuses also outside energy-related topics. Regarding those latter, the DT was studied to forecast components failures [42], traffic management [43] and autonomous guide [44]. NASA was a precursor of the adoption of DT to enhance transport efficiency and safety [45]. In terms of energy-related investigation, in [46], the authors analysed a part of the railways system as a high-power demanding load in the power grids. During the work, a DT model to perform a proper monitoring and control of operations was modelled. This approach, oriented to grid management, was adopted also in smaller cases addressing the issue of EV charging. The DT was used in [47] to perform a smart battery charging strategy to extend the battery lifetime, and similarly in [48], the same issue was assessed. Differently in [49], the focus was moved towards the grid perspective, analysing the possible auxiliary services that can be offered by the batteries to this latter. The methodology proposed in this work comprises an architecture for a DT in the domain of the high-voltage battery system and then to offer digital services for various stakeholders. Nevertheless, to reach a fully functional DT, this model needs to be implemented in a cloud computing environment which is not well defined yet. With a comprehensive approach, the authors in [50] have undertaken an ambitious investigation where a DT of an extensive portion of the city with all physical components was made. For the model, a total of 14,000 buildings including all locations of activities such as parking lots, transformers, middle-voltage lines, low-voltage lines of the distribution grid, and shops and homes were geo-referenced and modelled. Additionally, to correctly evaluate the population and mobility, also the city's inhabitants and commuters are modelled using agent-based simulations. Finally, this innovative method has been demonstrated using measurement data. Indeed, a system so defined can help in many ways in the city planning, management and resiliency, but it relies on a massive database system.

\section{CONCLUSIONS}

The paper reviewed the state of the art in terms of the use of the DT approach and concept applied to SESs. It firstly explained the particular application to SESs and the difficulties to such a wide and omni-comprehensive approach, and then it focused on the most interesting sectors and features of such systems. In conclusion, it can be stated that the powerful advantages offered by the DT concept are clear. Several barriers that hinder the spread of this concept can still be found, and they vary from technical, mainly connected to big data and communication protocols, to economic but especially in a lack of regulation and a clear market around the concept of demand response programmes. This uncertain and unclear framework led to many studies that developed different versions of DTs that can theoretically work in different frameworks based on different algorithms. Nevertheless, more specific DT can be found in single buildings or in other sectors such as the industrial.

Another common feature that has been noticed is that even though the DT concept is applied in several different studies regarding smart grid and SES, the keyword 'digital twin' is not always used. This signals the need for a further effort for interdisciplinary projects since, mostly from the energy/electricity sector, this term is not very well-known while it is always used by electronic/information professionals.

\section{REFERENCES}

[1] Mai, T.T., Jadun, P., Logan, J.S., McMillan, C.A., Muratori, M., Steinberg, D.C., Vimmerstedt, L.J., Haley, B., Jones, R. \& Nelson, B., Electrification Futures Study: Scenarios of Electric Technology Adoption and Power Consumption for the United States. United States: N., 2018. https://doi.org/10.2172/1459351 
[2] Philipp, G. \& Singaravel, S., Component-based machine learning for performance prediction in building design. Applied Energy, 228, pp. 1439-1453, 2018. https://doi. org/10.1016/j.apenergy.2018.07.011

[3] Grieves, M. \& Vickers, J., Digital twin: Mitigating unpredictable, undesirable emergent behavior in complex systems. In: Kahlen F.J., Flumerfelt, S., Alves, A. (eds.) Transdisciplinary Perspectives on Complex Systems. Springer, Cham, 2017. https://doi. org/10.1007/978-3-319-38756-7_4

[4] Barricelli, B.R., Casiraghi E. \& Fogli, D., A survey on digital twin: Definitions, characteristics, applications, and design implications. In IEEE Access, 7, pp. 167653-167671, 2019. https://doi.org/10.1109/ACCESS.2019.2953499

[5] Kong, T., Hu, T., Zhou, T. \& Ye, Y., Data construction method for the applications of workshop digital twin system. Journal of Manufacturing Systems, 58, Part B, pp. 323 328, 2021. https://doi.org/10.1016/j.jmsy.2020.02.003

[6] IEA, Smart Grids, IEA, Paris, 2020. https://www.iea.org/reports/smart-grids

[7] ENTSO-E , Digital Report: The cyber-physical system for the energy transition, ENTSO-E , 2019. https://www.entsoe.eu/news/2019/11/18/cyber-meets-the-physicalgrid-entso-e-s-digital-report-is-out/

[8] Blosch, M. \& Fenn, J., Understanding Gartner's Hype Cycles, Gartner, 2018.

[9] Brosinsky, C., Westermann, D. \& Krebs, R., Recent and prospective developments in power system control centers: Adapting the digital twin technology for application in power system control centers. 2018 IEEE International Energy Conference (ENERGYCON), pp. 1-6, 2018. https://doi.org/10.1109/ENERGYCON.2018.8398846

[10] Zheng, Y., Yang, S. \& Cheng, H., An application framework of digital twin and its case study. J Ambient Intell Human Comput, 10, pp. 1141-1153, 2019. https://doi. org/10.1007/s12652-018-0911-3

[11] Atalay, M. \& Angin, P., A digital twins approach to smart grid security testing and standardization. 2020 IEEE International Workshop on Metrology for Industry 4.0 \& IoT, pp. 435-440, 2020. https://doi.org/10.1109/MetroInd4.0IoT48571.2020.9138264

[12] Bellocchi, S., De Iulio, R., Guidi, G., Manno, M., Nastasi, B., Noussan, M., ... Roberto, R., Analysis of smart energy system approach in local alpine regions - A case study in northern italy. Energy, 202, 2020. https://doi.org/10.1016/j.energy.2020.117748

[13] Guelpa, E., Bischi, A., Verda, V., Chertkov, M. \& Lund, H., Towards future infrastructures for sustainable multi-energy systems: A review. Energy, 184, pp. 2-21, 2019. https://doi.org/10.1016/j.energy.2019.05.057

[14] Snijders, R., Pileggi, P., Broekhuijsen, J., Verriet, J., Wiering, M. \& Kok, K., Machine learning for digital twins to predict responsiveness of cyber-physical energy systems. Paper presented at the 8th Workshop on Modeling and Simulation of Cyber-Physical Energy Systems, MSCPES 2020 - Proceedings, 2020. https://doi.org/10.1109/ MSCPES49613.2020.9133695

[15] Park, H., Byeon, G., Son, W., Jo, H., Kim, J. \& Kim, S., Digital twin for operation of microgrid: Optimal scheduling in virtual space of digital twin. Energies, 13(20), 2020. https://doi.org/10.3390/en13205504

[16] Sittón-Candanedo, I., Alonso, R. S., García, Ó., Muñoz, L. \& Rodríguez-González, S., Edge computing, iot and social computing in smart energy scenarios. Sensors (Switzerland), 19(15), 2019. https://doi.org/10.3390/s19153353

[17] Zhou, M., Yan J. \& Feng, D., "Digital twin framework and its application to power grid online analysis. In CSEE Journal of Power and Energy Systems, 5(3), pp. 391-398, September 2019. https://doi.org/10.17775/CSEEJPES.2018.01460 
[18] Jain, P., Poon, J., Singh, J. P., Spanos, C., Sanders, S. R. \& Panda, S. K., A digital twin approach for fault diagnosis in distributed photovoltaic systems. In IEEE Transactions on Power Electronics, 35(1), pp. 940-956, January 2020. https://doi.org/10.1109/ TPEL.2019.2911594

[19] Ganesh, S., Perilla, A., Torres, J.R., Palensky, P. \& van der Meijden, M., Validation of EMT digital twin models for dynamic voltage performance assessment of $66 \mathrm{kV}$ offshore transmission network. Appl. Sci., 11, p. 244, 2021. https://doi.org/10.3390/ app11010244

[20] Wagner, T., Mehlmann, G. \& Richter, M., Application of the digital twin concept for a distribution network. PESS 2020; IEEE Power and Energy Student Summit, 2020, pp. 1-5.

[21] Feng, Z., et al., Online assessment of flexibility on active distribution network planning through digital twin. 2020 2nd International Conference on Electrical, Control and Instrumentation Engineering (ICECIE), pp. 1-6, 2020. https://doi.org/10.1109/ ICECIE50279.2020.9309627

[22] Feng, Z., Wu, Y., Gao, H. \& Zhu, S., Digital twin framework for ADN flexible resources assessment. 2020 IEEE Eurasia Conference on IOT, Communication and Engineering (ECICE), pp. 209-212, 2020. https://doi.org/10.1109/ECICE50847.2020.9302004

[23] Saad, A., Faddel, S., Youssef, T. \& Mohammed, O.A., On the implementation of IoT-based digital twin for networked microgrids resiliency against cyber attacks. In IEEE Transactions on Smart Grid, 11(6), pp. 5138-5150, November 2020. https://doi. org/10.1109/TSG.2020.3000958

[24] Francisco, A., Neda, M. \& Taylor, J.E., Smart city digital twin-enabled energy management: Toward real-time urban building energy. Journal of Management in Engineering, 36(2), March 2020. https://doi.org/10.1061/(ASCE)ME.1943-5479.0000741

[25] Ruohomäki, T., Airaksinen, E., Huuska, P., Kesäniemi, O., Martikka, M. \& Suomisto, J., Smart city platform enabling digital twin. 2018 International Conference on Intelligent Systems (IS), pp. 155-161, 2018. https://doi.org/10.1109/IS.2018.8710517

[26] Nastasi, B., Hydrogen policy, market and R\&D project. In Calise, F., D' Accadia, M.D., Santerelli, M., Lanzini, A., Ferrero, D., (eds.), Solar Hydrogen Production. Elsevier: Cambridge, MA, USA.

[27] Bowen, W., Guobin, Z., Huizhi, W., Jin, X. \& Kui, J., Multi-physics-resolved digital twin of proton exchange membrane fuel cells with a data-driven surrogate model. Energy and AI, 1, 2020, https://doi.org/10.1016/j.egyai.2020.100004

[28] Jia-Lin, K., Chien-Cheng, W., David Shan-Hill, W., Shi-Shang, J. \& Chun-Hsiu, W., Digital twin model and dynamic operation for a plant-scale solid oxide fuel cell system. Journal of the Taiwan Institute of Chemical Engineers, 118, pp. 60-67, 2021. https:// doi.org/10.1016/j.jtice.2021.01.001

[29] He, B. \& Bai, K.J., Digital twin-based sustainable intelligent manufacturing: A review. Adv. Manuf., 9, pp. 1-21, 2021. https://doi.org/10.1007/s40436-020-00302-5

[30] García, Ó., Alonso, R.S., Prieto, J. \& Corchado, J.M., Energy efficiency in public buildings through context-aware social computing. Sensors (Switzerland), 17(4), 2017. https://doi.org/10.3390/s17040826

[31] Ejaz, W., Naeem, M., Shahid, A., Anpalagan A. \& Jo, M., Efficient energy management for the internet of things in smart cities. In IEEE Communications Magazine, 55(1), pp. 84-91, January 2017. https://doi.org/10.1109/MCOM.2017.1600218CM

[32] Barbierato, L., et al., A distributed IoT infrastructure to test and deploy real-time demand response in smart grids. In IEEE Internet of Things Journal, 6(1), pp. 11361146, February 2019. https://doi.org/10.1109/JIOT.2018.2867511 
[33] Zhou, S. \& Zhang, L., Smart home electricity demand forecasting system based on edge computing. 2018 IEEE 9th International Conference on Software Engineering and Service Science (ICSESS), pp. 164-167, 2018, https://doi.org/10.1109/ ICSESS.2018.8663894

[34] Agostinelli, S., Cumo, F., Guidi, G. \& Tomazzoli, C. Cyber-physical systems improving building energy management: Digital twin and artificial intelligence. Energies, 14, p. 2338, 2021. https://doi.org/10.3390/en14082338

[35] Sittón-Candanedo, I., Alonso, R. S., García, Ó., Muñoz, L. \& Rodríguez-González, S., Edge computing, iot and social computing in smart energy scenarios. Sensors (Switzerland), 19(15), 2019. https://doi.org/10.3390/s19153353

[36] Agostinelli, S., Cumo, F., Guidi, G. \& Tomazzoli, C., The potential of digital twin model integrated with artificial intelligence systems. Paper presented at the Proceedings - 2020 IEEE International Conference on Environment and Electrical Engineering and 2020 IEEE Industrial and Commercial Power Systems Europe, EEEIC / I and CPS Europe 2020, 2020. https://doi.org/10.1109/EEEIC/ICPSEurope49358.2020.9160810

[37] Davila Delgado, J.M. \& Oyedele, L., Digital twins for the built environment: Learning from conceptual and process models in manufacturing. Advanced Engineering Informatics, 49, 2021. https://doi.org/10.1016/j.aei.2021.101332

[38] Shahinmoghadam, M., Natephra, W. \& Motamedi, A., BIM- and IoT-based virtual reality tool for real-time thermal comfort assessment in building enclosures. Building and Environment, 199, 2021. https://doi.org/10.1016/j.buildenv.2021.107905

[39] Desogus, G., Quaquero, E., Rubiu, G., Gatto, G. \& Perra, C., Bim and iot sensors integration: A framework for consumption and indoor conditions data monitoring of existing buildings. Sustainability (Switzerland), 13(8), 2021. https://doi.org/10.3390/ su13084496

[40] Zhu, J. \& Wu, P., A common approach to geo-referencing building models in industry foundation classes for bim/gis integration. ISPRS International Journal of Geo-Information, 10(6), 2021. https://doi.org/10.3390/ijgi10060362

[41] Zhu, J. \& Wu, P., Towards effective bim/gis data integration for smart city by integrating computer graphics technique. Remote Sensing, 13(10), 2021. https://doi.org/10.3390/ rs 13101889

[42] Bhatti, G., Mohan, H. \& Singh, R.R., Towards the future of smart electric vehicles: Digital twin technology. Renewable and Sustainable Energy Reviews, 141, 2021. https:// doi.org/10.1016/j.rser.2021.110801

[43] Kumar, S.A.P., Madhumathi, R. \& Chelliah, P.R., et al., A novel digital twin-centric approach for driver intention prediction and traffic congestion avoidance. J Reliable Intell Environ, 4, pp. 199-209, 2018. https://doi.org/10.1007/s40860-018-0069-y

[44] Bottani, E., Cammardella, A., Murino, T. \& Vespoli B., From the cyber-physical system to the digital twin: The process development for behaviour modelling of a cyber guided vehicle in M2M logic, 2017.

[45] Glaessgen, E.H. \& Stargel, D.S., The digital twin paradigm for future NASA and U.S. air force vehicles. Paper for the 53rd Structures, Structural Dynamics, and Materials Conference: Special Session on the Digital Twin. https://doi.org/10.2514/6.20121818

[46] Ahmadi, M., Kaleybar, H.J., Brenna, M., Castelli-Dezza F. \& Carmeli, M.S., Adapting digital twin technology in electric railway power systems. 2021 12th Power Electronics, Drive Systems, and Technologies Conference (PEDSTC), pp. 1-6, 2021. https://doi. org/10.1109/PEDSTC52094.2021.9405876 
[47] Ali, M.U., Zafar, A., Nengroo, S.H., Hussain, S., Alvi, M.J. \& Kim, H.J., Towards a smarter battery management system for electric vehicle applications: A critical review of lithium-ion battery state of charge estimation. Energies, 2019.

[48] Li, W., Kwiecien, M., Badeda, J, Jöst, D., Schulte, D. \& Sauer, D., Digital twin for battery systems: Cloud battery management system with online state-of-charge and state-of-health estimation. J Energy Storage, 30, p. 101557, 2020. https://doi.1016/j. est.2020.101557

[49] Merkle, L., Segura, A. S., Torben Grummel, J. \& Lienkamp, M., Architecture of a digital twin for enabling digital services for battery systems. 2019 IEEE International Conference on Industrial Cyber Physical Systems (ICPS), pp. 155-160, 2019. https://doi. org/10.1109/ICPHYS.2019.8780347

[50] Pagani, M., Korosec, W., Chokani, N. \& Abhari, R.S., User behaviour and electric vehicle charging infrastructure: An agent-based model assessment. Applied Energy, 254, 2019. https://doi.org/10.1016/j.apenergy.2019.113680 